\title{
BILAN DES INTRODUCTIONS RÉCENTES D'AMPHIBIENS ET DE REPTILES DANS LES MILIEUX AQUATIQUES CONTINENTAUX DE FRANCE MÉTROPOLITAINE.
}

\section{P. HAFFNER}

Muséum National d'Histoire Naturelle, Institut d'Ecologie et de Gestion de la Biodiversité, Service du Patrimoine Naturel, 57 rue Cuvier, 75231 Paris Cedex 05, France.

\section{RÉSUMÉ}

En France métropolitaine, on dénombre actuellement 36 amphibiens et 33 reptiles se reproduisant régulièrement. A deux exceptions près, les amphibiens de la faune française sont aquatiques. En revanche, seules quatre espèces de reptiles (2 tortues et 2 serpents) fréquentent très régulièrement ou exclusivement les milieux aquatiques.

Les introductions en milieux aquatiques effectuées depuis le début du siècle ne concernent qu'une vingtaine d'espèces d'amphibiens ou de reptiles. Le discoglosse peint (Discoglossus pictus) et la grenouille taureau (Rana catesbeiana) sont les deux seules espèces étrangères dont l'introduction a conduit à une naturalisation. La tortue de "Floride" (Trachemys scripta elegans) pourrait bien suivre prochainement la même voie. Certaines espèces françaises ont, par contre, été introduites avec succès en métropole, hors de leurs aires d'indigénat.

Les causes connues de ces introductions sont liées à des opérations à but économique (commerce), à caractère socioculturel (loisirs) ou à fondement scientifique (expériences). Ces opérations n'ont cependant généralement pas pour but l'introduction volontaire d'une espèce dans le milieu naturel, celle-ci résultant plutôt de négligences. Certaines introductions involontaires ont pu aussi avoir pour origine un transport passif (par exemple, par voie maritime).

Des conséquences négatives de ces introductions sont suspectées, mais ne sont généralement pas démontrées. Elles peuvent s'inscrire dans les catégories suivantes : compétition avec une espèce autochtone, prédation excessive sur une ou plusieurs espèces autochtones, pollution génétique, introduction de maladies ou de parasites.

\section{ASSESSMENT OF RECENT INTRODUCTIONS OF AMPHIBIANS AND REPTILES IN INLAND AQUATIC SYSTEMS IN METROPOLITAN FRANCE.}

\begin{abstract}
In Metropolitan France, 36 regularly breeding amphibians and 33 regularly breeding reptiles are presently recorded. With 2 exceptions, the amphibians of the French fauna are aquatic. In contrast, only 4 reptiles ( 2 turtles and 2 snakes) may be found very frequently or only in aquatic systems.
\end{abstract}

Since the beginning of the century, only about twenty amphibian and reptile species were introduced into aquatic systems. The painted frog (Discoglossus pictus) and the bullfrog (Rana catesbeiana) are the only foreign species whose introduction led to naturalization. The red-eared turtle (Trachemys scripta elegans) might follow in the near future. Moreover, in Metropolitan France, some French species have been successfully transferred outside their natural range. 
The known reasons for these introductions are linked to economic operations (trade) or to socio-cultural (leisure) or scientific (experiment) processes. However, the introduction of species into a natural habitat has often been due to carelessness rather than to a wilful operation. Some accidental introductions may also have been due to passive transport (e.g. by sea).

Negative outcomes to these introductions are suspected, but have generally not been demonstrated. They could fit in the following groups: competition with native species, too heavy predation on one or several native species, genetic pollution, introduction of diseases or parasites.

\section{INTRODUCTION}

A quelques exceptions près (espèces consommées ou utilisées comme animaux d'agrément), les amphibiens et les reptiles ont rarement joué un rôle économique prépondérant. Ils n'ont donc fait l'objet que de déplacements limités, tant en nombre d'espèces qu'en nombre d'individus, et les opérations d'introductions volontaires sont rares. Par contre, leur petite taille et leur tendance à se réfugier dans le premier trou venu les rendent susceptibles d'être transportés involontairement avec divers chargements. Ainsi, par exemple, le lézard des ruines (Podarcis sicula), originaire d'Italie et des îles adjacentes, a été retrouvé jusqu'en Amérique du Nord et certains gekkonidés ont colonisé presque toutes les régions tropicales du globe. Nous nous limiterons, dans les lignes qui suivent, aux introductions ayant eu lieu depuis le début du siècle dans les milieux aquatiques de France métropolitaine, qu'elles aient ou non été suivies d'acclimatation ou de naturalisation. Seront également évoquées les espèces initialement présentes en France et introduites ailleurs en métropole, hors de l'aire d'indigénat.

\section{PRÉSENTATION DES AMPHIBIENS ET DES REPTILES DE FRANCE MÉTROPOLITAINE}

En métropole, on dénombre maintenant 36 espèces d'amphibiens reproducteurs (11 urodèles, 25 anoures). Seules deux espèces se sont affranchies totalement des milieux aquatiques: la salamandre de Lanza (Salamandra lanzai) et le spélerpès brun (Hydromantes strinatii). Toutes les autres espèces ont besoin de la présence d'un point d'eau au moins pour le développement de leurs larves. La plupart y déposent aussi leurs pontes. Les amphibiens français hibernent généralement à terre, mais passent aussi parfois la mauvaise saison dans la vase des mares ou des etangs (certains individus de grenouilles rousses Rana temporaria, par exemple). Les amphibiens sont donc omniprésents dans les milieux aquatiques continentaux (eaux stagnantes surtout) et y jouent souvent un rôle prépondérant, aussi bien en tant que prédateurs qu'en tant que proies.

Les reptiles (33 espèces se reproduisant régulièrement en France, dont 3 tortues, 18 lézards et 12 serpents) sont beaucoup moins représentés dans les milieux aquatiques. Deux tortues (l'emyde lépreuse Mauremys leprosa et la cistude d'Europe Emys orbicularis) et deux serpents (la couleuvre vipérine Natrix maura et, dans une moindre mesure, la couleuvre à collier $N$. natrix) sont inféodés aux eaux douces. Les tortues aquatiques et la couleuvre vipérine peuvent rester de longues heures dans l'eau et s'en éloignent rarement. La couleuvre à collier, bien qu'elle soit également très liée aux zones humides, peut aussi se rencontrer dans des milieux plus secs. Toutes ces espèces pondent à terre. Les tortues aquatiques peuvent hiberner dans l'eau. Par contre, les couleuvres passent toujours la mauvaise saison à terre.

\section{LES ESPĖCES AYANT FAIT L'OBJET D'INTRODUCTIONS}

\section{Les amphibiens}

Seuls les anoures ont, semble-t-il, fait l'objet d'introductions dans les milieux aquatiques continentaux de métropole. Un urodèle, le triton crêté d'Italie (Triturus carnifex), a toutefois été 
introduit il y a une trentaine d'années en Suisse (canton de Genève), non loin de la frontière avec la France (GROSSENBACHER, 1988). II a été signalé, depuis, dans la partie française du lac Léman (ZUIDERWIJK, 1989). Pour mémoire, rappelons qu'un urodèle non aquatique des Alpes du sud, le spélerpès brun, a été introduit dans une grotte d'Ariège.

\section{Le discoglosse peint (Discoglossus pictus)}

L'existence d'une population de discoglosse peint n'est connue en métropole que depuis 1906 (WINTREBERT, 1908). Sa découverte a eu lieu à Banyuls-sur-Mer (Pyrénées-Orientales). Bien que l'hypothèse d'une introduction ait été avancée à cette époque, celle-ci n'a pas été reprise par les auteurs suivants. En particulier, KNOEPFFLER (1962) croyait en l'existence d'une aire de répartition continue de l'espèce, depuis l'Afrique du Nord jusque dans les PyrénéesOrientales, via la Péninsule lbérique. Il faudra attendre une révision du genre par B. LANZA en 1986, pour que l'identité subspécifique de cette population ( $D$. p. algirus) soit connue. L'aire de répartition de cette sous-espèce s'étend de l'Algérie à la Tunisie (LANZA, 1989). D'autre part, il s'avère que les populations ibériques appartiennent à une autre espèce, le discoglosse de Galgano (D. galganoi).

L'introduction, dont les causes sont inconnues, a probablement eu lieu à la fin du siècle dernier ou au tout début de ce siècle, dans la région même de Banyuls-sur-Mer. Depuis, l'aire de répartition de cette population s'est étendue assez rapidement sur la côte catalane, dans toutes les directions où un passage était possible (MARTENS et VEITH, 1987). Ce schéma, montrant une extension d'aire à partir d'un " foyer ", est en accord avec l'hypothèse d'une introduction ponctuelle. Le fait que ce mouvement d'expansion se poursuive actuellement suggère une introduction récente.

Le discoglosse peint a, depuis, fait l'objet d'introductions volontaires infructueuses dans les bassins de la Seine et de la Loire. Il semble être naturalisé au Jardin des Plantes, à Paris. Cette espèce est protégée en France.

\section{La grenouille taureau (Rana catesbeiana)}

Originaire d'Amérique du Nord, la grenouille taureau a èté introduite sur plusieurs continents. En Europe, elle est naturalisée en Italie (ALBERTINI et LANZA, 1987), aux Pays-Bas (STUMPEL, 1992) et peut-être aussi en Espagne. La naturalisation de cette grenouille en métropole est assez récente, bien que des tentatives infructueuses aient été faites à la fin du siècle dernier (DUBOIS, 1983). La première mention écrite signalant la naturalisation de l'espèce (région de Bordeaux) date de 1990 (TOURATIER, 1992a). En fait, cette population est issue d'individus relâchés à la fin des années 80 . La grenouille taureau n'est pas protégée en France.

\section{La grenouille rieuse (Rana ridibunda)}

En raison de la grande difficulté à déterminer les différentes espèces de grenouilles " vertes ", la répartition exacte de la grenouille rieuse est encore mal connue. II apparait toutefois que son aire de répartition soit constituée de populations isolées, qui ont certainement pour origine des introductions. Seules les populations de l'est de la France et autour du lac Léman pourraient être autochtones (NEVEU, 1989). II est probable que des introductions répétées se soient produites tout au long de ce siècle. L'origine des individus est multiple; les principales zones à partir desquelles se sont opérées les importations étant l'ex-Yougoslavie, la Turquie et l'Egypte (DUBOIS, 1983). Cette espèce est protégée en France.

\section{Autres amphibiens ayant fait l'objet d'introductions ponctuelles}

La rainette méridionale (Hyla meridionalis), présente dans le Midi méditerranéen, a été introduite sur les îles d'Hyères (KNOEPFFLER, 1961). Elle y est maintenant naturalisée. Des discoglosses sardes (Discoglossus sardus), en provenance des îles d'Hyères, ont été introduits dans le massif des Maures en 1955. L'espèce ne s'y est pas maintenue (KNOEPFFLER, 1962). Ces deux espèces sont protégées en France. 


\section{Les reptiles}

Les introductions de reptiles dans les milieux aquatiques continentaux de France métropolitaine concernent essentiellement des tortues.

\section{La tortue de "Floride " (Trachemys scripta elegans)}

La tortue de "Floride " est une sous-espèce de la trachémyde écrite ( $T$. scripta), tortue aquatique dont la vaste aire de répartition s'etend naturellement depuis le Venezuela jusqu'au nord des États-Unis. La trachémyde écrite a, par ailleurs, été largement introduite de par le monde. En métropole, on ne rencontre que $T$. s. elegans, improprement appelée tortue de "Floride ", car elle est précisément absente de cet état. La tortue de "Floride " fait l'objet d'importations continues depuis l'interdiction de la commercialisation des jeunes aux ÉtatsUnis, en 1975, pour des motifs sanitaires (risques de Salmonellose). Elle a été observée dans pratiquement tous les départements français. De rares indices de reproduction ont été obtenus, montrant ainsi que l'espèce est non seulement acclimatée, mais qu'elle est aussi en voie de naturalisation (SERVAN, comm. pers.). Cette tortue n'est pas protégée en France.

\section{Autres tortues aquatiques introduites dans les milieux aquatiques de France continentale}

Pratiquement toutes les espèces importées peuvent être trouvées dans la nature. Ont été ainsi signalées : la tortue hargneuse (Chelydra serpentina), la tortue alligator (Macroclemys temminckii), le trionyx épineux (Apalone spinifera), le trionyx de Chine (Pelodiscus sinensis), l'emyde mutique (Mauremys mutica) et la graptémyde pseudogéographique (Graptemys pseudogeographica). Le triony $x$ de Chine et l'emyde mutique sont originaires d'Asie. Les autres espèces proviennent d'Amérique du Nord. Elles n'ont été trouvées qu'occasionnellement dans la nature et ne semblent pas présenter un risque élevé de naturalisation, si le volume de leur importation reste faible et leur prix d'achat moins attractif que celui de la tortue de "Floride". Ces tortues ne sont pas protégées.

Les deux espèces autochtones, la cistude d'Europe (Emys orbicularis) et l'emyde lépreuse (Mauremys leprosa), ont également fait l'objet d'introductions hors de leurs aires d'indigénat (SERVAN, 1989 ; COLIN, 1992). Ces introductions n'ont, semble-t-il, pas été suivies de naturalisations. La cistude d'Europe et l'emyde lépreuse étant maintenant protégées en France, ce genre d'opération ne devrait plus se renouveler. On signale pourtant encore, de temps à autre, des individus de ces espèces hors de leurs aires d'indigénat (voir par exemple COLIN, 1992).

\section{La couleuvre vipérine (Natrix maura)}

L'aire de répartition de ce serpent, très lié aux eaux douces, va de la Péninsule Ibérique à la Suisse et à l'Italie. L'espèce habite aussi le Maghreb. Elle est commune dans les deux tiers sud de la métropole. Présente sur certaines îles méditerranéennes (Baléares, Sardaigne), la couleuvre vipérine était inconnue en Corse jusqu'à une date récente. En 1980 et en 1985, elle a été observée en deux régions de cette île : Sartène ( $400 \mathrm{~m}$ d'altitude) et Aléria (à proximité de la côte). Les prospections herpétologiques assidues, dont la Corse avait fait précédemment l'objet, ainsi que le caractère assez peu discret de cette espèce rendent peu probable une présence ancienne restée inaperçue (FONS et al., 1991). L'arrivée de l'espèce se serait effectuée en deux fois, comme le suggère la grande distance entre les deux points d'observation. Les individus introduits pourraient venir de la Sardaigne toute proche. II reste toutefois difficile de savoir s'il s'agit d'introductions volontaires ou involontaires. La naturalisation de l'espèce en Corse n'est pas établie. L'espèce est protégée en France, ce qui devrait en principe limiter les opérations d'introduction volontaire.

\section{La couleuvre tessellée (Natrix tessellata)}

Cette couleuvre, originaire du sud-est de l'Europe et d'Asie, est encore plus aquatique que la couleuvre vipérine. Trouvée à plusieurs reprises sur notre territoire, probablement après s'être échappée d'élevages, elle ne semble jamais s'être acclimatée. 
Présente naturellement en Suisse (cantons de Ticino et Posciavo), la couleuvre vipérine a récemment été introduite dans ce même pays entre Lausanne et Montreux (HOFER et DUSEJ, 1995). Cette opération pourrait conduire à une installation de l'espèce dans les territoires français adjacents. Toutefois, elle n'a pas encore atteint le bassin de Genève et ce risque est, pour l'instant, limité (GROSSENBACHER, comm. pers.).

\section{CAUSES DES INTRODUCTIONS}

II n'est pas toujours possible de déterminer les causes des introductions de reptiles ou d'amphibiens. La discrétion de ces animaux, leur taille modeste, ainsi que la relative indifférence dont fait preuve le public à leur égard, font qu'il existe peu d'informations écrites sur ces événements. Les introductions ne sont parfois constatées que de nombreuses années après qu'elles se soient produites, de telle sorte qu'une longue enquête est nécessaire pour reconstituer les circonstances dans lesquelles elles se sont effectuées. Les origines des introductions peuvent être regroupées dans les catégories suivantes (MAURIN et al., 1994) : les opérations à but économique visant essentiellement le profit, les opérations à caractère socioculturel avec un objectif d'agrément ou de loisir, et celles à fondement scientifique ou écologique. Toutes ces opérations, qui s'accompagnent d'importations d'animaux, ne visent pas nécessairement à introduire des espèces dans le milieu naturel. Mais, bien souvent, des animaux finissent par s'échapper ou bien sont relâchés, de telle sorte qu'une acclimatation suivie d'une naturalisation peut se produire. A ces causes, il faudrait ajouter les introductions involontaires résultant du transport passif d'animaux.

\section{Opérations à but économique}

La grenouille rieuse a fait l'objet d'un commerce intense. Celle-ci est, en effet, plus grosse que les grenouilles vertes (Rana $\mathrm{kl}$. esculenta et $R$. lessonae) et la grenouille rousse ( $R$. temporaria) dont on consomme traditionnellement les cuisses. Ce commerce a largement contribué à la dispersion de l'espèce sur le territoire métropolitain français. La fuite d'individus ainsi que l'abandon de certains animaux à la suite par exemple de la fermeture d'un élevage, ont conduit à la naturalisation de l'espèce.

Ce commerce a également été une des causes de la dispersion de la grenouille taureau à travers le monde.

\section{Opérations à caractère socioculturel}

Les introductions de tortues aquatiques (en particulier, de la tortue de " Floride") sont essentiellement liées à leur importation en tant qu'animaux de compagnie. Les motivations ayant conduit à ces importations sont donc tout autant d'ordre économique que socioculturel, une certaine mode de la tortue s'étant développée ces dernières années. Les tortues de "Floride" sont vendues alors qu'elles ne mesurent que $5 \mathrm{~cm}$ de long. La beauté de leur livrée ainsi que leur petite taille les rendent très attractives, en particulier pour les enfants. De plus, leur prix n'est pas élevé, ce qui finit par convaincre les parents. En quelques années, elles peuvent atteindre $25 \mathrm{~cm}$ de long. Elles sont alors souvent considérées trop encombrantes par leurs propriétaires qui, désireux de s'en débarrasser, les relâchent dans la nature.

Dans le cas de la grenouille taureau, il semble bien que quelques individus aient été importés de Louisiane par un conseiller général, uniquement pour étonner son entourage avec cette espèce spectaculaire. En effet, la grenouille taureau peut atteindre $2 \mathrm{~kg}$. La naturalisation n'était sûrement pas voulue.

\section{Opérations à fondement scientifique ou écologique}

La grenouille rieuse a largement été utilisée dans les laboratoires universitaires. Des individus échappés de captivité ou relâchés ont parfois rejoint le milieu naturel et s'y sont naturalisés (NEVEU, 1989). 
L'introduction infructueuse du discoglosse sarde dans le massif des Maures par KNOEPFFLER en 1955 a été réalisée dans le cadre d'une expérience in natura, visant à étudier la compétition entre cette espèce et d'autres amphibiens et reptiles méditerranéens (KNOEPFFLER, 1962).

A l'étranger, les amphibiens et les reptiles ont parfois été introduits lors d'expériences de lutte biologique (crapaud de la canne à sucre Bufo marinus, dans de nombreuses régions tropicales du monde, afin de lutter contre les insectes nuisibles; téju commun Tupinambis teguixin, sur l'île brésilienne de Fernando de Noronha, pour lutter contre les rats; etc.). II ne semble pas que de telles motivations aient conduit à des tentatives d'introductions d'amphibiens ou de reptiles dans les milieux aquatiques de France métropolitaine, hormis quelques expériences très localisées avec le crapaud commun Bufo bufo (LESCURE, comm. pers.).

\section{Introductions involontaires suite à un transport passif}

II est possible, bien que cela ne soit pas prouvé, que le discoglosse peint ainsi que la couleuvre vipérine en Corse soient arrivés avec des chargements transportés par des bateaux. On peut, par exemple, remarquer que les relations maritimes entre Port-Vendres et l'Algérie étaient importantes à l'époque présumée de l'introduction du discoglosse peint dans la région de Banyuls-sur-Mer (WINTREBERT, 1908). Ce mécanisme d'introduction, bien connu par exemple chez les gekkonidés ou certains lacertidés, intervient certainement souvent chez les amphibiens et les reptiles dont les tailles sont réduites. II explique, la plupart du temps, l'installation récente d'une espèce sur une île.

\section{CONSÉQUENCES SUR LES ÉCOSYSTÈMES ET LES ESPÈCES}

Les impacts des introductions d'amphibiens et de reptiles en milieux aquatiques ont été peu étudiés en France. Les expériences étrangères dans ce domaine (MOYLE, 1973; ALBERTINI et LANZA, 1987 ; KUPFERBERG, 1993) nous permettent d'émettre des hypothèses sur les conséquences possibles de l'introduction d'une espèce donnée. Une telle comparaison doit cependant rester prudente, car chaque introduction possède ses particularités liées, entre autres, aux caractéristiques du milieu d'accueil, à l'espèce introduite et aux biocénoses en place.

Les conséquences des introductions sont multiples. Elles peuvent conduire à une perturbation du fonctionnement des écosystèmes récepteurs, menant parfois à la disparition d'une ou de plusieurs espèces. Ces impacts peuvent se faire ressentir même si l'espèce introduite n'est pas naturalisée. Les tortues aquatiques, en particulier, vivent très longtemps. Si un apport continu d'individus s'effectue dans le milieu naturel, comme dans le cas de la tortue de "Floride", les conséquences peuvent être identiques à celles induites par une population naturalisée.

\section{Compétition avec une espèce autochtone}

La compétition interspécifique peut prendre plusieurs formes. Dans le cas des amphibiens et des reptiles, c'est la compétition alimentaire qui est le plus souvent évoquée.

La grenouille rieuse et la grenouille taureau peuvent potentiellement entrer en compétition avec des espèces occupant des niches proches, en particulier avec les autres espèces de grenouilles "vertes " qui sont de tailles inférieures (HONEGGER, 1978 ; DUBOIS, 1983). Cette compétition peut se produire au stade adulte, mais aussi au stade larvaire comme cela a pu être montré expérimentalement entre la grenouille taureau et la grenouille de Boyle Rana boylei (KUPFERBERG, 1993). Par contre, d'après VEITH et MARTENS (1987), le discoglosse peint n'entre pas en compétition avec d'autres amphibiens autochtones en Catalogne. 
Une prochaine compétition est très probable entre la tortue de "Floride" et la cistude d'Europe. Une telle compétition est également à craindre entre le triton crêté d'Italie (Triturus carnifex) et le triton crêté ( $T$. cristatus), ainsi qu'entre la couleuvre tessellée et la couleuvre vipérine. L'introduction de la couleuvre tessellée sur les bords du lac Léman a conduit à une réduction des effectifs de la couleuvre vipérine, sans toutefois avoir conduit à l'extinction de cette dernière (GROSSENBACHER, comm. pers.).

\section{Prédation excessive sur une ou plusieurs espèces}

La grenouille taureau est bien connue pour sa voracité. Elle peut ingérer toute une gamme de vertébrés (poissons, amphibiens, tortues aquatiques, petits mammifères, jeunes oiseaux). En particulier, la prédation par la grenouille taureau d'amphibiens autochtones en voie de régression a été mise en évidence à la suite d'analyses de contenus stomacaux en Californie ou en Italie, deux régions où celle-ci a été introduite (MOYLE, 1973 ; ALBERTINI et LANZA, 1987 ; TOURATIER, 1992b). Par ailleurs, les pêcheurs d'Aquitaine accusent la grenouille taureau de consommer les alevins et d'être responsable de la diminution des poissons (TOURATIER, 1992a). Cette prédation est cependant certainement limitée, la grenouille taureau n'étant pas adaptée à chasser sous l'eau (NEVEU, comm. pers.). La grenouille rieuse, introduite en Suisse, serait également responsable de la régression d'amphibiens autochtones qu'elle consommerait (HONEGGER, 1978). Les grenouilles du genre Rana consomment les discoglosses et joueraient un rôle primordial dans la distribution des espèces de ce genre. Ceci expliquerait, en partie, l'échec de l'introduction du discoglosse sarde dans les Maures (KNOEPFFLER, 1962). Remarquons, toutefois, qu'aucune étude n'a été faite in natura sur l'impact réel de la prédation de la grenouille taureau ou de la grenouille rieuse sur les espèces autochtones. Or, l'observation simultanée de la régression d'une espèce autochtone et de sa prédation par une espèce introduite ne prouve pas l'existence d'un lien de cause à effet.

La couleuvre vipérine est un prédateur inhabituel pour les espèces aquatiques de Corse. Ces dernières pourraient donc constituer des proies faciles. Ainsi, certaines populations de poissons ou d'amphibiens pourraient diminuer, suite à la présence de la couleuvre vipérine. Celle-ci est en particulier connue pour consommer les têtards du discoglosse sarde sur l'île du Levant (KNOEPFFLER, 1961, 1962). Ce discoglosse est également présent en Corse. Mais surtout, il existe sur cette île une espèce endémique, le discoglosse corse ( $D$. montalentii), dont le statut est assez précaire car ses populations sont très localisées et d'effectifs faibles. Toutes les tortues aquatiques introduites peuvent également potentiellement conduire à la régression de certaines de leurs proies autochtones. On observe, par exemple, la diminution du nombre d'individus de grenouilles ou de crapauds dans les petites mares où la tortue est présente (SERVAN, comm. pers.).

\section{Pollution génétique}

Les risques de pollution génétique par la grenouille rieuse sont souvent évoqués (DUBOIS, 1983). La proximité génétique de cette espèce avec les autres grenouilles "vertes" est telle que son introduction pourrait mener à des modifications du patrimoine génétique de certaines populations, avant même que la systématique de ces espèces ne soit totalement éclaircie. En effet, même en France, l'inventaire et la description des différents taxons appartenant au complexe des grenouilles "vertes" ne sont pas terminés (voir, par exemple, CROCHET et al., 1995).

De telles modifications génétiques ont également pu affecter la cistude d'Europe. Trois sous-espèces seraient présentes en métropole. Or, l'homme est à l'origine de nombreuses translocations d'individus d'une région à une autre, depuis quelques siècles.

\section{Introduction de maladies ou de parasites}

Au niveau des écosystèmes aquatiques, aucun problème sanitaire lié à l'introduction d'amphibiens ou de reptiles n'a été constaté en métropole. Cependant, des études devraient être effectuées dans ce domaine, en particulier pour la grenouille taureau (TOURATIER, 1992b), 
mais aussi pour la tortue de "Floride". On ne connait pas, par exemple, les conséquences sanitaires sur le milieu naturel de l'introduction de tortues de "Floride " ayant séjourné plusieurs années dans des élevages.

\section{CONCLUSION}

Les opérations récentes d'introduction d'amphibiens et de reptiles dans les milieux aquatiques continentaux, hormis celles relatives à la tortue de "Floride ", sont peu fréquentes et ne concernent qu'un nombre limité d'espèces. Par aileurs, elles n'ont généralement pas pour objectif la naturalisation des espèces concernées. Toutefois, la naturalisation récente de la grenouille taureau et celle de la tortue de "Floride " risquent d'occasionner des perturbations dans les écosystèmes aquatiques, pouvant aller jusqu'à la disparition d'espèces autochtones. II convient donc de prendre d'urgence toutes les mesures nécessaires pour limiter leur propagation, telle par exemple l'interdiction de les importer. La loi 95-101 du 2 février 1995, relative au renforcement de la protection de l'environnement, prévoit des mesures pour prévenir toute introduction d'espèce exogène dans le milieu naturel. Elle devrait donc jouer un rôle primordial, en particulier en ce qui concerne les amphibiens et reptiles "à problèmes ".

Enfin, les conséquences réelles des introductions d'amphibiens ou de reptiles sont mal connues et souvent hypothétiques. Des études et des suivis scientifiques devraient être développés en métropole, afin de déterminer les impacts réels de celles-ci, en particulier en ce qui concerne la grenouille taureau et la tortue de "Floride".

\section{REMERCIEMENTS}

Je remercie vivement, pour leur aide précieuse et leurs remarques constructives, Roger BOUR (MNHN), Kurt GROSSENBACHER (Naturhistorisches Museum Bern), Jean LESCURE (MNHN), André NEVEU (INRA) et Jean SERVAN (MNHN).

\section{BIBLIOGRAPHIE}

ALBERTINI G., LANZA B., 1987. Rana catesbeiana Shaw, 1802 in Italy. Alytes, 6 (3-4), 117-129.

COLIN F., 1992. Sur la présence de 3 espèces de Tortues aquatiques en Eure-et-Loir. Soc. Amis Mus. Chartres Nat. Eure-et-Loir : Bull., 11, 45-48.

CROCHET P.A., DUBOIS A., OHLER A., TUNNER H., 1995. Rana (Pelophylax) ridibunda Pallas, 1771, Rana (Pelophylax) perezi Seoane, 1885 and their associated klepton (Amphibia, Anura) : morphological diagnosis and description of a new taxon. Bull. Mus. Natl. Hist. Nat., Paris, 4e sér., 17 (1-2), 11-30.

DUBOIS A., 1983. A propos de cuisses de grenouilles. Alytes, 2 (3), 69-111.

FONS R., SAINT GIRONS H., SALOTTI M., CHEYLAN M., CLARA J.P., 1991. Contribution à la faune herpétologique des îles méditerranéennes: présence de la Couleuvre vipérine Natrix maura (Reptilia, Colubridae) en Corse. Bonn. Zool. Beitr., 42 (2), 181-186.

GROSSENBACHER K., 1988. Atlas de distribution des Amphibiens de Suisse. Documenta Faunistica Helvetiae $n^{\circ} 8$, Centre Suisse de Cartographie de la Faune et Ligue Suisse pour la Protection de la Nature, Bâle, $208 \mathrm{p}$.

HOFER U., DUSEJ G., 1995. Distribution and status of the reptiles in Switzerland, a preliminary report. In LLORENTE et al., eds., Scientia Herpetologica, 297-302, Asociación Herpetológica Española, Barcelona.

HONEGGER R.E., 1978. Amphibiens et Reptiles menacés en Europe. Collection Sauvegarde de la Nature, 15, Conseil de l'Europe, Strasbourg, $127 \mathrm{p}$. 
KNOEPFFLER L.P., 1961. Les Batraciens et principalement le genre Discoglossus dans les îles méditerranéennes. In ANONYME, Colloques internationaux du Centre National de la Recherche Scientifique $n^{\circ} X C I V$, Le peuplement des îles méditerranéennes et le problème de l'insularité, Banyuls-sur-Mer, 21-27 septembre 1959, 159-161, Centre National de la Recherche Scientifique, Paris.

KNOEPFFLER L.P., 1962. Contribution à l'étude du genre Discoglossus (Amphibiens, Anoures). Thèse de Doctorat d'Université (30 mai 1961), Faculté des Sciences, Paris, $96 \mathrm{p}$.

KUPFERBERG S.J., 1993. Bullfrogs (Rana catesbeiana) invade a northern California river : a plague or species coexistence ? Froglog, 7, 4.

LANZA B., 1989. Discoglossus pictus. In CASTANET J., GUYETANT R., coord., Atlas de répartition des Amphibiens et Reptiles de France, 63, Société Herpétologique de France, Paris.

MARTENS H., VEITH M., 1987. Considerations on origin and chronology of Discoglossus pictus Otth, 1837 in the eastern Pyrenees. In GELDER (VAN) J.J., STRIJBOSCH H., BERGERS P.J.M., eds., Proceedings of the 4th Ordinary General Meeting of the Societas Europaea Herpetologica, 267-269, Faculty of Sciences of Nijmegen, Nijmegen.

MAURIN H., HAFFNER P., KEITH P., 1994. Bilan des introductions et réintroductions de vertébrés sauvages en France métropolitaine depuis le début du siècle. In BODSON L., ed., Des animaux introduits par l'homme dans la faune d'Europe, Colloques d'histoire des connaissances zoologiques $n^{\circ} 5$ : journée d'étude du 20 mars 1993, 79-104, Université de Liège, Liège.

MOYLE P.B., 1973. Effects of introduced Bullfrogs, Rana catesbeiana, on the native frogs of the San Joaquin Valley, California. Copeia, (1), 18-22.

NEVEU A., 1989. Rana ridibunda. In CASTANET J., GUYETANT R., coord., Atlas de répartition des Amphibiens et Reptiles de France, 91, Société Herpétologique de France, Paris.

SERVAN J., 1989. Emys orbicularis. In CASTANET J., GUYETANT R., coord., Atlas de répartition des Amphibiens et Reptiles de France, 107, Société Herpétologique de France, Paris.

STUMPEL A.H.P., 1992. Successful reproduction of introduced bullfrogs Rana catesbeiana in northwestern Europe : a potential threat to indigenous amphibians. Biological Conservation, 60, 61-62.

TOURATIER L., 1992a. Première apparition en France (Région Aquitaine) d'une grenouille géante américaine : Rana catesbeiana, en voie d'acclimatement. Intérêt zoologique et impact éventuel sur l'environnement. Bull. Soc. Vet. Prat. France, 76 (4), 219-228.

TOURATIER L., 1992b. Similitudes et différences actuellement apparentes entre les Grenouilles taureaux (Rana catesbeiana) en voie de propagation en Italie et en France. Emergence des questions de santé publique vétérinaire. Bull. Soc. Vet. Prat. France, 76 (6-7), 349355.

VEITH M., MARTENS H., 1987. What's the part of Discoglossus pictus? Analysis of an ecological niche in a frog community. In GELDER (VAN) J.J., STRIJBOSCH H., BERGERS P.J.M., eds., Proceedings of the 4th Ordinary General Meeting of the Societas Europaea Herpetologica, 433-436, Faculty of Sciences of Nijmegen, Nijmegen.

WINTREBERT P., 1908. Présence à Banyuis-sur-Mer (Pyrénées-Orientales) du Discoglossus pictus Othh. Bull. Soc. Zool. Fr., 33, 54.

ZUIDERWIJK A., 1989. Triturus cristatus. In CASTANET J., GUYETANT R., coord., Atlas de répartition des Amphibiens et Reptiles de France, 45, Société Herpétologique de France, Paris. 